\title{
Anforderung an kleine und mittelständische Unternehmen des Landes Brandenburg zur Gründung eines Joint Ventures in der Volksrepublik China
}

\author{
Marén Schenk
}

China ist ein Zauberwort, welches schon immer die Phantasie und Meinungsbildung der Menschen angeregt hat.

Nach Schätzungen der Weltbank und anderer internationaler Organisationen zählt der chinesische Wirtschaftsraum, zumindest der Kaufkraft nach, in 15 bis 20 Jahren zu den stärksten Wirtschaftsregionen der Welt. Unternehmen aus aller Welt sind in diesem Zukunftsmarkt mittlerweile präsent, zum Jahresende 1996 erreichte die Zahl der ausländischen Unternehmen im Lande 140.000 mit insgesamt 17 Millionen Beschäftigten.

Diese Dynamik lockt Investoren aus Asien, Amerika und Europa. Jetzt werden die Märkte verteilt, jetzt finden Liberalisierung, Deregulierung und Marktöffnung statt. Es ist daher gut, daß die Entwicklung Chinas auch in Deutschland mehr Interesse und Aufmerksamkeit findet als noch vor ein paar Jahren. „Die Wettbewerbsfähigkeit Deutschlands wird nicht in Europa entschieden, sondern in Asien" (Lothar Späth, Aufsichtsratsvorsitzender der Jenoptik).

Viele deutsche Unternehmen haben ihre Chancen erkannt und sind heute an vielen Projekten beim Auf- und Ausbau der Infrastruktur (Telekommunikation, Verkehr, Logistik) sowie anderen Schwerpunktbranchen der Volksrepublik China wie Energiewirtschaft, Umwelttechnologien, Konsumgüterversorgung beteiligt. Dazu hat das neue Asienkonzept der Bundesregierung genauso beigetragen wie der Asien-Pazifik Ausschuß der Deutschen Wirtschaft. Ziel ist es, deutsche Unternehmen für ein stärkeres Engagement in der asiatisch-pazifischen Region zu gewinnen und die politischen und wirtschaftlichen Rahmenbedingungen für ein stärkerer Engagement asiatischer Partner in Deutschland zu verbessern. Viele große Unternehmen können auf eine langjährige Präsenz in China zurüickblicken. Siemens beispielsweise exportierte schon 1872 nach China und hat heute 40 Joint Venture dort, davon zwei WFOE (100\% Tochterunternehmen).

Ein Mittelständler riskiert jedoch möglicherweise bei einem ersten Schritt nach China seine Existenz. Bisher sind kleine deutsche Firmen überwiegend im Geleitzug von Großunternehmen in die asiatisch-pazifischen Länder gegangen, etwa als Unterauftragnehmer bei Großprojekten. Dieser Weg hat sich bewährt.
Meine Diplomarbeit soll kleinen und mittelständischen Unternehmen (KMU):

a) allgemeine Informationen über Marktbedingungen und Besonderheiten des chinesischen Marktes zuliefern,

b) ein Hilfsmittel darstellen, das KMU in die Lage versetzt, den Verfahrensweg zur Gründung von Joint Venture kennen- und anwenden zu lernen.

Die Diplomarbeit setzt sich aus zwei Teilen zusammen, einem praktischen und einem theoretischen Teil. Ein umfangreiches Literaturstudium und diverse Recherchen bildeten die Grundlage für den theoretischen Teil. Hier bin ich unter anderem auf die Wirtschaftsdaten Chinas, die Formen des Außenhandels in China sowie das Gründungsverfahren bei einem Joint Venture eingegangen.

Natïrlich war der praktische Teil für mich der interessanteste. Dazu wurden zehn brandenburgische Unternehmen, die in China tätig sind, befragt. Hierfür habe ich einen Fragebogen entwickelt, der als Leitfaden für die mündlichen Interviews benutzt wurde. Als Gesprächspartner wurde der Geschäftsfuihrer bzw. der zuständige Mitarbeiter gewählt. Die Interviews fanden vor Ort bei den jeweiligen Unternehmen statt.

Zum besseren Verständnis ist zu sagen, daß von den befragten neun KMU zwei Unternehmen Consulter sind, die keinen direkten Joint Venture Partner haben. Sieben KMU haben einen Joint Venture Partner in China. Von den sieben KMU hat aber nur ein Unternehmen ein bereits funktionstüchtiges Joint Venture. Die restlichen sechs KMU sind noch in der Gründungsphase und haben mit erheblichen Schwierigkeiten zu kämpfen.

Auf die wichtigste Frage - Welche Anforderungen muß Ihrer Meinung nach ein KMU erfüllen, um in China ein Joint Venture gründen zu können? - konnten nur wenige antworten, da nur zwei Unternehmen ein funktionsfähiges Joint Venture haben. Im folgenden sind einige Antworten der Unternehmer zusammengestellt.

Anforderungen an das Produkt:

- vor Ort feststellen, ob ein Markt für mein Produkt vorhanden ist, Absatzsicherung

- abchecken, wie hoch der Bedarf von staatliche Stellen ist, d. h. Absatzsicherung von staatlicher Seite oder Partner $=$ Kunde

- ständige Kontrolle bei Qualität 
Anforderungen an den Partner und Standort:

- Teilnahme an Unternehmerreisen von WFB und anderen Stellen, da man so Kontakte knïpfen kann und die Landesregierung dahinter steht

- Personal muß man ausbilden

- Englischkenntnisse sind zwingend

- Vertrauensperson ganz wichtig

Anforderungen an Management und Vertrieb:

- gründliche Informationsbeschaffung über den chinesischen Markt und positive Einstellung auf die kulturellen Unterschiede; gründliche Marktanalyse

- Studien am besten von Experten erstellen lassen, z. B. von erfahrenen Consultern

- Finanzierungsmodell vorlegen

- Dokumente müssen ins Englische übersetzt werden

Meine Recherchen haben ergeben, daß China für brandenburgische Unternehmen durchaus ein attraktiver Zielmarkt ist. Das betrifft vor allem Wirtschaftszweige, die moderne und innovative Technologien anbieten. Dies sind beispielsweise die Umwelttechnik, Telekommunikation und die Verkehrstechnik.

Zur Zeit ist die Zahl der brandenburgischen Unternehmen, die in China tätig sind, noch sehr gering. Daher müssen diese Unternehmen für die Chance, die der chinesische Markt Ihnen bietet, vorbereitet werden.

Das Joint Venture als Investitionsform ist meiner Meinung nach für KMU die beste Variante, da gerade kleine und mittlere Unternehmen auf den chinesischen Partner angewiesen sind.

Für die deutsche bzw. brandenburgische Wirtschaft bedeutet ein Engagement in China die Schaffung und Sicherung von Arbeitsplätzen. Erfahrungen deutscher Großunternehmen haben gezeigt, daß drei Arbeitsplätze in China einen Arbeitsplatz in Deutschland schaffen bzw. erhalten, z. B. durch die Zulieferung von Komponenten aus Deutschland.

Wer heute nicht anfängt, in China zu investieren, für den kann es morgen schon zu spät sein.

Die wichtigsten Bedingungen für ein Engagement in China lassen sich mit drei Buchstaben beschreiben: $\mathrm{P}-\mathrm{R}-\mathrm{C}$. Das bedeutet nicht People Republic of China, sondern Patience Relation Cash (Geduld - Beziehungen - Kapital).

\section{Verfasserin}

\section{Marén Schenk}

(4. Studienjahr, Betriebswirtschaftslehre)

Technische Fachhochschule Wildau

Bahnhofstraße

15745 Wildau 